Supporting information

for

\title{
Synthesis of Heavily Substituted 1,2-Amino Alcohols in Enantiomerically Pure Form
}

Noemí García-Delgado, Katamreddy Subba Reddy, Lluís Solà, Antoni Riera, Miquel A. Pericàs,

Xavier Verdaguer

Table of contents:

Complete experimental details for compounds 11a-d, 12a-d and 13a-d....................... S-2

${ }^{1} \mathrm{H}$ and ${ }^{13} \mathrm{C}$ NMR spectra for compounds $9, \mathbf{1 1 d}, \mathbf{1 2 d}, \mathbf{1 3 b}$ and 13d........................... S-11 
General Information. All reactions were carried out under an inert atmosphere. Dichlorometane was distilled under nitrogen from $\mathrm{CaH}_{2}$. Melting points have been determined by differential scanning calorimetry (DSC). Infrared spectra were recorded using $\mathrm{NaCl}$ film or $\mathrm{KBr}$ pellet techniques. ${ }^{1} \mathrm{H}$ chemical shifts are quoted relative to TMS and ${ }^{13} \mathrm{C}$ shifts relative to solvent signals. Carbons multiplicities have been assigned by distorsionless enhancement polarization transfer (DEPT) experiments. Commercial Jacobsen catalysts $(1 R, 2 R)-(-)-N, N^{\prime}$-Bis(3,5-di-tert-butylsalicylidene)-1,2cyclohexanediaminomanganese (III) chloride was used throughout the present study.

\section{General procedure for the olefin epoxidation (Scheme S-1)}
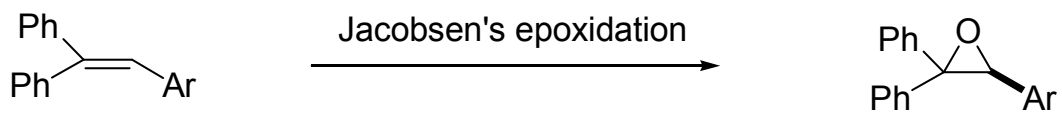

Scheme S-1

A solution of $\mathrm{NaClO}$ ( previously tritated and adjusted to $\mathrm{pH}=11.3$ ) was added dropwise to a solution of the olefin, $(R, R)$-Jacobsen's catalyst and 4-phenylpyridine $\mathrm{N}$-oxide in anhydrous $\mathrm{CH}_{2} \mathrm{Cl}_{2}$ at $0{ }^{\circ} \mathrm{C}$. When the reaction ceased to evolve, as determined by TLC, the solution was filtered with Celite ${ }^{\circledR}$. The filtrate was washed with brine, the organic layer was dried $\left(\mathrm{MgSO}_{4}\right)$ and concentrated under vacuum. The residue was purified by column chromatography on silica gel/ $\mathrm{NEt}_{3} 2.5 \% \mathrm{v} / \mathrm{v}$ (hexane and hexane/ethyl acetate $0.5 \%$ ).

(S)-3-(4-methoxyphenyl)-2,2-diphenyloxirane (11a). Prepared according to general procedure from a solution of 1-(2-(4-methoxyphenyl)-1-phenylvinyl)benzene (5.66 g, $19.77 \mathrm{mmol}),(R, R)$-Jacobsen's catalyst $(0.61 \mathrm{~g}, 0.97 \mathrm{mmol})$, 4-phenylpyridine N-oxide $(0.72 \mathrm{~g}, 4.21 \mathrm{mmol})$ in $20 \mathrm{~mL}$ of anhydrous $\mathrm{CH}_{2} \mathrm{Cl}_{2}$ and a solution of $\mathrm{NaClO}(0.57 \mathrm{M}, \mathrm{pH}=11.3,70 \mathrm{~mL})$ for 3.5 hours. The desired product was 
obtained as a yellow oil in $96 \%$ yield $(5.75 \mathrm{~g}, 19.01 \mathrm{mmol})$. The product was recristalized from diethyl ether to afford a crystalline solid of $>99 \%$ ee. $\mathrm{Mp}=96{ }^{\circ} \mathrm{C} ;[\alpha]_{\mathrm{D}}+69.7\left(\mathrm{c} 1.04, \mathrm{CHCl}_{3}\right) ;{ }^{1} \mathrm{H}-\mathrm{NMR}(400$ $\left.\mathrm{MHz}, \mathrm{CDCl}_{3}\right) \delta 7.37-7.19(\mathrm{~m}, 10 \mathrm{H}), 6.93(\mathrm{~d}, J=9 \mathrm{~Hz}, 2 \mathrm{H}), 6.67(\mathrm{~d}, J=9 \mathrm{~Hz}, 2 \mathrm{H}), 4.26(\mathrm{~s}, 1 \mathrm{H}), 3.73(\mathrm{~s}$, 3H) ppm; ${ }^{13} \mathrm{C}-\mathrm{NMR}\left(100 \mathrm{MHz}, \mathrm{CDCl}_{3}\right) \delta 159.2(\mathrm{C}), 141.1(\mathrm{C}), 135.9(\mathrm{C}), 129.2(\mathrm{CH}), 128.3(\mathrm{CH})$, $128.0(\mathrm{CH}), 127.9(\mathrm{CH}), 127.7(\mathrm{CH}), 127.5(3)(\mathrm{C}), 127.5(1)(\mathrm{CH}), 126.2(\mathrm{CH}), 113.2(\mathrm{CH}), 68.5(\mathrm{C})$, $68.0(\mathrm{CH}), 55.1\left(\mathrm{CH}_{3}\right)$; IR (KBr) $v_{\max } .3028,2860,1516,1447,696 \mathrm{~cm}^{-1}$; MS (ESI-TOF) m/z: 303 $\left[\left(\mathrm{M}^{+}+1\right), 100 \%\right]$; Anal. Cald for $\mathrm{C}_{21} \mathrm{H}_{18 \mathrm{O}_{2}}$ : C 83.42, H 6.00 Found C 83.26, H 6.03; HPLC Daicel CHIRALCEL-ODR, MeOH, $0.5 \mathrm{~mL} / \mathrm{min}, \lambda=254 \mathrm{~nm}, t_{\mathrm{R}}(S)=12.6 \mathrm{~min}, t_{\mathrm{R}}(R)=15.6 \mathrm{~min}$.

(S)-3-(4-fluorophenyl)-2,2-diphenyloxirane (11b). Prepared according to general procedure from a solution of 1-(2-(4-fluorophenyl)-1-phenylvinyl)benzene (4.44 g, $16.20 \mathrm{mmol}),(R, R)$-Jacobsen's catalyst $(0.50 \mathrm{~g}, 0.79 \mathrm{mmol})$, 4-phenylpyridine N-oxide $(0.59 \mathrm{~g}, 3.48 \mathrm{mmol})$ in $15 \mathrm{~mL}$ of anhydrous $\mathrm{CH}_{2} \mathrm{Cl}_{2}$ and a solution of $\mathrm{NaClO}(0.57 \mathrm{M}, \mathrm{pH}=11.3,57 \mathrm{~mL})$ for 6 hours. The desired product was obtained as a yellow solid in $92 \%$ yield $(4.31 \mathrm{~g}, 14.84 \mathrm{mmol})$. The product was recristalized from hexane to afford a crystalline solid of $>99 \%$ ee. $\mathrm{Mp}=125{ }^{\circ} \mathrm{C} ;[\alpha]_{\mathrm{D}}+66.8\left(\mathrm{c} 1.07, \mathrm{CHCl}_{3}\right) ;{ }^{1} \mathrm{H}-\mathrm{NMR}$ (400 MHz, $\left.\mathrm{CDCl}_{3}\right) \delta$ 7.37-7.17 (m, 10H), 7.05-6.95 (m, 2H), $6.83(\mathrm{t}, J=8 \mathrm{~Hz}, 2 \mathrm{H}), 4.30(\mathrm{~s}, 1 \mathrm{H}), \mathrm{ppm}$; ${ }^{13} \mathrm{C}-\mathrm{NMR}\left(100 \mathrm{MHz}, \mathrm{CDCl}_{3}\right) \delta 162.6\left(\mathrm{C}, J_{F}=245 \mathrm{~Hz}\right), 141.0(\mathrm{C}), 135.9(\mathrm{C}), 131.6\left(\mathrm{C}, J_{F}=3 \mathrm{~Hz}\right)$, $129.5(\mathrm{CH}), 128.7(\mathrm{CH}), 128.7\left(\mathrm{CH}, J_{F}=8 \mathrm{~Hz}\right), 128.3(\mathrm{CH}), 128.2(\mathrm{CH}), 128.0(\mathrm{CH}), 126.6(\mathrm{CH})$, $115.1\left(\mathrm{CH}, J_{F}=21 \mathrm{~Hz}\right), 68.9(\mathrm{C}), 67.8(\mathrm{CH}) \mathrm{ppm}$; IR $(\mathrm{KBr}) v_{\max .} 3085,1509,1447,762,702 \mathrm{~cm}^{-1}$; HRMS (ESI-TOF) calcd for $\mathrm{C}_{20} \mathrm{H}_{16} \mathrm{FO}$ : 291.1185, found 291.1198; Anal. Cald for $\mathrm{C}_{20} \mathrm{H}_{16} \mathrm{FO}$ : C 82.74, H 5.21 Found C 82.71, H 5.22; HPLC Daicel CHIRALCEL-ODR, MeOH/solution $\mathrm{NaClO}_{4}$ 0.5M 90:10, $0.5 \mathrm{~mL} / \mathrm{min}, \lambda=254 \mathrm{~nm}, t_{\mathrm{R}}(R)=17.1 \mathrm{~min}, t_{\mathrm{R}}(S)=18.5 \mathrm{~min}$.

(S)-3-biphenyl-2,2-diphenyloxirane (11c). Prepared according to general procedure from a solution of 2-biphenyl-4-yl-1,1-diphenyl-ethene (1.00 g, $3.01 \mathrm{mmol}),(R, R)$-Jacobsen's catalyst $(93 \mathrm{mg}, 0.15$ mmol), 4-phenylpyridine $\mathrm{N}$-oxide $(109 \mathrm{mg}, 0.64 \mathrm{mmol})$ in $2.5 \mathrm{~mL}$ of anhydrous $\mathrm{CH}_{2} \mathrm{Cl}_{2}$ and a solution 
of $\mathrm{NaClO}(0.57 \mathrm{M}, \mathrm{pH}=11.3,10.6 \mathrm{~mL})$ for 2.5 hours. The desired product was obtained as a paleyellow solid in $86 \%$ yield $(0.91 \mathrm{~g}, 2.61 \mathrm{mmol}), 86 \%$ ee. The product was recristalized from hexane to afford a crystalline solid of $>99 \%$ ee. $\mathrm{Mp}=146{ }^{\circ} \mathrm{C} ;[\alpha]_{\mathrm{D}}+62.33\left(\mathrm{c} 1.00, \mathrm{CHCl}_{3}\right) ;{ }^{1} \mathrm{H}-\mathrm{NMR}(400 \mathrm{MHz}$, $\left.\mathrm{CDCl}_{3}\right) \delta 7.52(\mathrm{~d}, J=8 \mathrm{~Hz}, 2 \mathrm{H}), 7.40-7.20(\mathrm{~m}, 14 \mathrm{H}), 7.10(\mathrm{~d}, J=8 \mathrm{~Hz} .3 \mathrm{H}), 4.35(\mathrm{~s}, 1 \mathrm{H}) \mathrm{ppm} ;{ }^{13} \mathrm{C}-\mathrm{NMR}$ $\left(100 \mathrm{MHz}, \mathrm{CDCl}_{3}\right) \delta 140.9(\mathrm{C}), 140.5(\mathrm{C}), 140.3(\mathrm{C}), 135.7(\mathrm{C}), 134.5(\mathrm{C}), 129.2(\mathrm{CH}), 128.7(\mathrm{CH})$, $128.3(\mathrm{CH}), 127.9(\mathrm{CH}), 127.8(\mathrm{CH}), 127.6(\mathrm{CH}), 127.3(\mathrm{CH}), 127.2(\mathrm{CH}), 126.9(\mathrm{CH}), 126.4(\mathrm{CH})$, $126.3(\mathrm{CH}), 68.7(\mathrm{C}), 68.0(\mathrm{CH})$ ppm; IR (KBr) $v_{\max .} 3424,3027,1488,1448,700 \mathrm{~cm}^{-1}$; MS (CI-NH$\left.)_{3}\right)$ m/z: $348\left[\left(\mathrm{M}^{+}+1\right), 100 \%\right]$; Anal. Cald for $\mathrm{C}_{26} \mathrm{H}_{20} \mathrm{O}: \mathrm{C}$ 89.62, H 5.79 Found C 89.46, H 5.73; HPLC Daicel CHIRALCEL-ODR. MeOH, $0.5 \mathrm{~mL} / \mathrm{min}, \lambda=254 \mathrm{~nm}, t_{\mathrm{R}}(R)=16.9 \mathrm{~min}, t_{\mathrm{R}}(S)=24.7 \mathrm{~min}$.

(S)-3-(3,5-dimethylphenyl)-2,2-diphenyloxirane (11d). Prepared according to general procedure from a solution of 1-(2-(3,5-dimethylphenyl)-1-phenylvinyl)benzene (600 mg, $2.11 \mathrm{mmol}),(R, R)$ Jacobsen's catalyst (66 mg, $0.10 \mathrm{mmol})$, 4-phenylpyridine N-oxide (77 mg, $0.45 \mathrm{mmol})$ in $3 \mathrm{~mL}$ of anhydrous $\mathrm{CH}_{2} \mathrm{Cl}_{2}$ and a solution of $\mathrm{NaClO}(0.50 \mathrm{M}, \mathrm{pH}=11.3,8.5 \mathrm{~mL})$ for 16 hours. The desired product was obtained as a colorless oil in $44 \%$ yield $(277 \mathrm{mg}, 0.92 \mathrm{mmol}), 70 \%$ ee. $[\alpha]_{\mathrm{D}}+28.05(\mathrm{c}$ 0.98, $\left.\mathrm{CHCl}_{3}\right) ;{ }^{1} \mathrm{H}-\mathrm{NMR}\left(400 \mathrm{MHz}, \mathrm{CDCl}_{3}\right) \delta$ 7.39-7.18 (m, 10H), $6.78(\mathrm{~s}, 1 \mathrm{H}), 6.63(\mathrm{~s}, 1 \mathrm{H}), 4.24(\mathrm{~s}$, 1H), 2.14 (s, 6H) ppm; ${ }^{13} \mathrm{C}-\mathrm{NMR}\left(100 \mathrm{MHz}, \mathrm{CDCl}_{3}\right) \delta 141.3(\mathrm{C}), 137.3$ (C), 136.1 (C), $135.4(\mathrm{C}), 129.4$ $(\mathrm{CH}), 129.3(\mathrm{CH}), 128.4(\mathrm{CH}), 127.9(\mathrm{CH}), 127.8(\mathrm{CH}), 127.6(\mathrm{CH}), 127.5(\mathrm{CH}), 124.8(\mathrm{CH}), 68.7(\mathrm{C})$, $68.2(\mathrm{CH}), 21.2\left(\mathrm{CH}_{3}\right)$ ppm; IR (film) $v_{\max }$ 3028, 2917, 1605, 1493, $1447 \mathrm{~cm}^{-1}$; MS (EI) m/z: 300 [(M+), 9.12\%]; HRMS (EI) calcd for $\mathrm{C}_{22} \mathrm{H}_{20} \mathrm{O}$ : 300.1514, found 300.1505; HPLC Daicel CHIRALCEL-OD, Hexane/i-PrOH 95:5, $0.5 \mathrm{~mL} / \mathrm{min}, \lambda=254 \mathrm{~nm}, t_{\mathrm{R}}(S)=8.4 \mathrm{~min}, t_{\mathrm{R}}(R)=10.6 \mathrm{~min}$. 


\section{General procedure for the epoxide ring opening (Scheme S-2)}
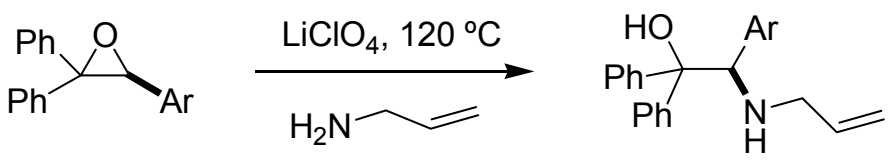

Scheme S-2

A pressure tube equipped with a magnetic stir bar was charged with the appropiate epoxide, allylamine and $\mathrm{LiClO}_{4}$. The suspension was heated to $120{ }^{\circ} \mathrm{C}$ until the epoxide was consumed as determined by TLC. The resulting solution solidifed when cooled to room temperature. The crude was disolved with $\mathrm{CH}_{2} \mathrm{Cl}_{2}$ and the organic layer was washed successively with brine. The organics were then dried $\left(\mathrm{MgSO}_{4}\right)$ and removed together with the amine under vacuum. The desired $\beta$-aminoalcohol was obtained in a quantitative yield and purified by silica gel chromatography (hexane/ethyl acetate) if necessary.

(R)-2-(allylamino)-2-(4-methoxyphenyl)-1,1-diphenylethanol (12a). Prepared according to general procedure from $(S)$-3-(4-methoxyphenyl)-2,2-diphenyloxirane $11 \mathbf{a}(0.250 \mathrm{~g}, 0.83 \mathrm{mmol}), \mathrm{LiClO}_{4}(0.176$ $\mathrm{g}, 1.65 \mathrm{mmol})$ and allylamine $(0.62 \mathrm{~mL}, 8.27 \mathrm{mmol})$ in a $15 \mathrm{~mL}$ pressure tube. The suspension was then stirred at $120^{\circ} \mathrm{C}$ overnight. The pure product was obtained as a white solid in $99 \%$ yield $(0.290 \mathrm{~g}, 0.81$ mmol). $\mathrm{Mp}=129^{\circ} \mathrm{C} ;[\alpha]_{\mathrm{D}}+159.9\left(c 1.00, \mathrm{CHCl}_{3}\right) ;{ }^{1} \mathrm{H}-\mathrm{NMR}\left(400 \mathrm{MHz}, \mathrm{CDCl}_{3}\right) \delta 7.69(\mathrm{~d}, J=7 \mathrm{~Hz}$, 2H), 7.40-6.97 (m, 10H), $6.65(\mathrm{~d}, J=9 \mathrm{~Hz}, 2 \mathrm{H}), 5.78(\mathrm{~m}, 1 \mathrm{H}), 5.04(\mathrm{~d}, J=9 \mathrm{~Hz}, 1 \mathrm{H}), 4.98(\mathrm{dd}, J=17$ $\left.\mathrm{Hz}, J^{\prime}=1 \mathrm{~Hz}, 1 \mathrm{H}\right), 4.63(\mathrm{~s}, 1 \mathrm{H}), 4.30\left(\right.$ broad s, 1H), $3.73(\mathrm{~s}, 3 \mathrm{H}), 3.09\left(\mathrm{dd}, J=14 \mathrm{~Hz}, J^{\prime}=5 \mathrm{~Hz}, 1 \mathrm{H}\right)$, $2.95\left(\mathrm{dd}, J=14 \mathrm{~Hz}, J^{\prime}=7 \mathrm{~Hz}, 1 \mathrm{H}\right), 1.56(\operatorname{broad} \mathrm{s}, 1 \mathrm{H}) \mathrm{ppm} ;{ }^{13} \mathrm{C}-\mathrm{NMR}\left(100 \mathrm{MHz}, \mathrm{CDCl}_{3}\right) \delta 158.6(\mathrm{C})$, $145.9(\mathrm{C}), 144.0(\mathrm{C}), 136.2(\mathrm{CH}), 130.5(\mathrm{CH}), 129.4(\mathrm{CH}), 128.4(\mathrm{CH}), 127.3(\mathrm{CH}), 127.1(\mathrm{CH}), 126.6$ $(\mathrm{CH}), 126.1(\mathrm{CH}), 126.1(\mathrm{CH}), 116.3(\mathrm{C}), 112.8(\mathrm{CH}), 79.9(\mathrm{C}), 67.5(\mathrm{CH}), 55.1\left(\mathrm{CH}_{3}\right), 49.3\left(\mathrm{CH}_{2}\right)$ ppm; IR (KBr) $v_{\max .} 3443,2927,1611,1513 \mathrm{~cm}^{-1}$; MS $\left(\mathrm{CI}-\mathrm{NH}_{3}\right) \mathrm{m} / z: 360.1\left[(\mathrm{M}+1)^{+}, 8.7 \%\right], 175.8$ $\left[\left(\mathrm{C}_{11} \mathrm{H}_{14} \mathrm{NO}\right)^{+}, 100 \%\right]$; Anal. Cald for $\mathrm{C}_{24} \mathrm{H}_{25} \mathrm{NO}_{2}$ : C 80.19, H 7.01, N 3.90 Found C 80.40, H 6.93, N 3.74 . 
(R)-2-(allylamino)-2-(4-fluorophenyl)-1,1-diphenylethanol (12b). Prepared according to general procedure from $(S)$-3-(4-fluorophenyl)-2,2-diphenyloxirane $\mathbf{1 1 b}(250 \mathrm{mg}, 0.86 \mathrm{mmol}), \mathrm{LiClO}_{4}(183 \mathrm{mg}$, $1.72 \mathrm{mmol})$ and allylamine $(0.65 \mathrm{~mL}, 8.61 \mathrm{mmol})$ in a $15 \mathrm{~mL}$ pressure tube. The suspension was then stirred at $120^{\circ} \mathrm{C}$ overnight. The product was obtained as a yellow solid in $95 \%$ yield (285 $\mathrm{mg}, 0.8$ mmol). $\mathrm{Mp}=133^{\circ} \mathrm{C} ;[\alpha]_{\mathrm{D}}+194.4\left(c 1.04, \mathrm{CHCl}_{3}\right) ;{ }^{1} \mathrm{H}-\mathrm{NMR}\left(400 \mathrm{MHz}, \mathrm{CDCl}_{3}\right) \delta 7.69\left(\mathrm{dd}, J=9 \mathrm{~Hz}, J^{\prime}\right.$ $=1 \mathrm{~Hz}, 2 \mathrm{H}), 7.40-6.80(\mathrm{~m}, 12 \mathrm{H}), 5.77(\mathrm{~m}, 1 \mathrm{H}), 5.05(\mathrm{~d}, J=10 \mathrm{~Hz}, 1 \mathrm{H}), 4.97(\mathrm{~d}, J=17 \mathrm{~Hz}, 1 \mathrm{H}), 4.17(\mathrm{~s}$, 1H), $3.09\left(\mathrm{dd}, J=14 \mathrm{~Hz}, J^{\prime}=5 \mathrm{~Hz}, 1 \mathrm{H}\right), 2.94\left(\mathrm{dd}, J=15 \mathrm{~Hz}, J^{\prime}=7 \mathrm{~Hz}, 1 \mathrm{H}\right), 1.60($ broad s, 2H) ppm; ${ }^{13} \mathrm{C}-\mathrm{NMR}\left(100 \mathrm{MHz}, \mathrm{CDCl}_{3}\right) \delta 162.0\left(\mathrm{C}, J_{F}=244 \mathrm{~Hz}\right), 145.7(\mathrm{C}), 143.7(\mathrm{C}), 135.9(\mathrm{CH}), 133.1\left(\mathrm{C}, J_{F}=\right.$ $3 \mathrm{~Hz}), 131.0\left(\mathrm{CH}, J_{F}=8 \mathrm{~Hz}\right), 128.6(\mathrm{CH}), 127.4(\mathrm{CH}), 127.3(\mathrm{CH}), 126.6(\mathrm{CH}), 126.3(\mathrm{CH}), 125.9$ $(\mathrm{CH}), 116.6\left(\mathrm{CH}_{2}\right), 114.2\left(\mathrm{CH}, J_{F}=21 \mathrm{~Hz}\right), 79.9(\mathrm{C}), 67.3(\mathrm{CH}), 49.3\left(\mathrm{CH}_{2}\right) \mathrm{ppm}$; IR $(\mathrm{KBr}) v_{\max .} 3432$, 3058, 2920, 1602, $1508 \mathrm{~cm}^{-1}$. MS $\left(\mathrm{CI}^{\left.-\mathrm{NH}_{3}\right)} \mathrm{m} / \mathrm{z}: 348.3\left[\left(\mathrm{M}^{+}+1\right), 65 \%\right], 163.9\left[\left(\mathrm{C}_{10} \mathrm{H}_{11} \mathrm{NF}^{+}\right), 100 \%\right]\right.$; Anal. Cald for $\mathrm{C}_{23} \mathrm{H}_{22} \mathrm{FNO}$ : C 79.51, H 6.38, N 4.03 Found C 79.24, H 6.29, N 3.91.

(R)-2-(allylamino)-2-biphenyl-1,1-diphenylethanol (12c). Prepared according to general procedure from (S)-3-biphenyl-2,2-diphenyloxirane 11c (150 mg, $0.45 \mathrm{mmol}), \mathrm{LiClO}_{4}(96 \mathrm{mg}, 0.90 \mathrm{mmol})$ and allylamine $(0.34 \mathrm{~mL}, 4.51 \mathrm{mmol})$ in a $15 \mathrm{~mL}$ pressure tube. The suspension was then stirred at $120^{\circ} \mathrm{C}$ for 3.5 days. The pure product was obtained as a white solid in $75 \%$ yield (137 $\mathrm{mg}, 0.3 \mathrm{mmol})$ after purification by silica gel. $\mathrm{Mp}=142{ }^{\circ} \mathrm{C} ;[\alpha]_{\mathrm{D}}+196.2\left(c\right.$ 1.02, $\left.\mathrm{CHCl}_{3}\right) ;{ }^{1} \mathrm{H}-\mathrm{NMR}\left(400 \mathrm{MHz}, \mathrm{CDCl}_{3}\right) \delta$ $7.72(\mathrm{~d}, J=7 \mathrm{~Hz}, 2 \mathrm{H}), 7.54(\mathrm{~d}, J=7 \mathrm{~Hz}, 2 \mathrm{H}), 7.42-7.25(\mathrm{~m}, 8 \mathrm{H}), 7.13(\mathrm{~m}, 4 \mathrm{H}), 7.03(\mathrm{~m}, 3 \mathrm{H}), 5.81(\mathrm{~m}$, 1H), $5.08(\mathrm{~d}, J=10 \mathrm{~Hz}, 1 \mathrm{H}), 5.02(\mathrm{~d}, J=18 \mathrm{~Hz}, 1 \mathrm{H}), 4.72(\mathrm{~s}, 1 \mathrm{H}), 4.32(\operatorname{broad} \mathrm{s}, 1 \mathrm{H}), 3.15(\mathrm{dd}, J=15$ $\left.\mathrm{Hz}, J^{\prime}=5 \mathrm{~Hz}, 1 \mathrm{H}\right), 3.01\left(\mathrm{dd}, J=14 \mathrm{~Hz}, J^{\prime}=7 \mathrm{~Hz}, 1 \mathrm{H}\right), 1.56(\operatorname{broad} \mathrm{s}, 1 \mathrm{H}) \mathrm{ppm} ;{ }^{13} \mathrm{C}-\mathrm{NMR}(100 \mathrm{MHz}$, $\left.\mathrm{CDCl}_{3}\right) \delta 146.2(\mathrm{C}), 144.2(\mathrm{C}), 141.1(\mathrm{C}), 140.2(\mathrm{C}), 137.0(\mathrm{C}), 136.5(\mathrm{CH}), 130.2(\mathrm{CH}), 129.0(\mathrm{CH})$, $128.9(\mathrm{CH}), 127.7(\mathrm{CH}), 127.6(\mathrm{CH}), 127.5(\mathrm{CH}), 127.3(\mathrm{CH}) ; 127.0(\mathrm{CH}), 126.6(\mathrm{CH}), 126.5(\mathrm{CH})$, $126.4(\mathrm{CH}), 116.8\left(\mathrm{CH}_{2}\right), 79.9(\mathrm{C}), 68.3(\mathrm{CH}), 49.8\left(\mathrm{CH}_{2}\right)$ ppm; IR $(\mathrm{KBr}) v_{\max .} 3394,3029,1597,1411$ $\mathrm{cm}^{-1}$; MS $\left(\mathrm{CI}-\mathrm{NH}_{3}\right) \mathrm{m} / z: 406.2\left[(\mathrm{M}+1)^{+}, 93.9 \%\right], 222.1\left[\left(\mathrm{C}_{16} \mathrm{H}_{16} \mathrm{~N}\right)^{+}, 100 \%\right]$; Anal. Cald for $\mathrm{C}_{29} \mathrm{H}_{27} \mathrm{NO}$ : C 85.89, H 6.71, N 3.45 Found C 86.20, H 6.37, N 3.35. 
(R)-2-(allylamino)-2-(3,5-dimethylphenyl)-1,1-diphenylethanol (12d). Prepared according to general procedure from (S)-3-(3,5-dimethylphenyl)-2,2-diphenyloxirane 11d (162 mg, $0.54 \mathrm{mmol}$ ), $\mathrm{LiClO}_{4}(114 \mathrm{mg}, 1.08 \mathrm{mmol})$ and allylamine $(0.4 \mathrm{~mL}, 5.39 \mathrm{mmol})$ in a $15 \mathrm{~mL}$ pressure tube. The suspension was then stirred at $120^{\circ} \mathrm{C}$ for 40 hours. The product was obtained as a white solid in $90 \%$ yield $(172 \mathrm{mg}, 0.48 \mathrm{mmol})$ after purification by silica gel. $\mathrm{Mp}=81-82{ }^{\circ} \mathrm{C} ;[\alpha]_{\mathrm{D}}+119.3\left(\mathrm{c} 1.00, \mathrm{CHCl}_{3}\right)$; ${ }^{1} \mathrm{H}-\mathrm{NMR}\left(400 \mathrm{MHz}, \mathrm{CDCl}_{3}\right) \delta 7.68(\mathrm{~d}, J=8 \mathrm{~Hz}, 2 \mathrm{H}), 7.38(\mathrm{t}, J=8 \mathrm{~Hz}), 7.27(\mathrm{t}, J=8 \mathrm{~Hz}, 1 \mathrm{H}), 7.13-$ $6.98(\mathrm{~m}, 5 \mathrm{H}), 6.76(\mathrm{~s}, 1 \mathrm{H}), 6.63(\mathrm{~s}, 2 \mathrm{H}), 5.80(\mathrm{~m}, 1 \mathrm{H}), 5.03(\mathrm{~m}, 2 \mathrm{H}), 4.57(\mathrm{~s}, 1 \mathrm{H}), 4.31$ (broad s, 1H), $3.12\left(\mathrm{dd}, J_{1}=14 \mathrm{~Hz}, J_{2}=5 \mathrm{~Hz}, 1 \mathrm{H}\right), 2.99\left(\mathrm{dd}, J_{1}=14 \mathrm{~Hz}, J_{2}=7 \mathrm{~Hz}, 1 \mathrm{H}\right), 2.14(\mathrm{~s}, 6 \mathrm{H}) \mathrm{ppm} ;{ }^{13} \mathrm{C}-\mathrm{NMR}$ $\left(100 \mathrm{MHz}, \mathrm{CDCl}_{3}\right) \delta 145.9(\mathrm{C}), 144.2(\mathrm{C}), 137.5(\mathrm{C}), 136.8(\mathrm{CH}), 136.5(\mathrm{C}), 128.9(\mathrm{CH}), 128.5(\mathrm{CH})$, $127.5(\mathrm{CH}), 127.3(\mathrm{CH}), 127.2(\mathrm{CH}), 126.9(\mathrm{CH}), 126.5(\mathrm{CH}), 126.3(\mathrm{CH}), 116.4\left(\mathrm{CH}_{2}\right), 80.0(\mathrm{C}), 68.4$ (CH), $49.8\left(\mathrm{CH}_{2}\right), 21.3\left(\mathrm{CH}_{3}\right)$ ppm; IR (KBr) v $\max .3471,2917,1602,1448,669 \mathrm{~cm}^{-1} ; \mathrm{MS}\left(\mathrm{CI}_{-} \mathrm{CH}_{4}\right) \mathrm{m} / z$ : $358.5\left[(\mathrm{M}+1)^{+}, 80.5 \%\right], 340.5\left[\left(\mathrm{C}_{25} \mathrm{H} 2{ }_{6} \mathrm{~N}\right)^{+}, 61.0 \%\right]$; HRMS $\left(\mathrm{CI}-\mathrm{CH}_{4}\right)$ : calcd for $\mathrm{C}_{25} \mathrm{H}_{27} \mathrm{NO}\left(\mathrm{M}+\mathrm{H}^{+}\right)$ 358.2171, found 358.2166.

General procedure for the amine deprotection (Scheme S-3) 


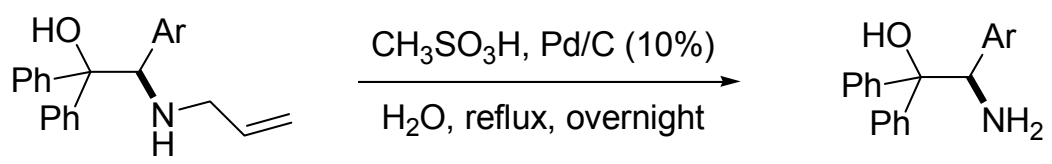

Scheme S-3

The appropiate $\beta$-aminoalcohol, methanesulfonic acid, palladium on charcoal $10 \%$ and water were added to a two-necked roundbottomed-flask. The mixture was heated under reflux overnight. A slow flow of argon passed throught the solution to aid removal of propionaldehyde. The solution was then basified with $\mathrm{NaOH}$ to $\mathrm{pH} 10$ and a white solid appears. Celite ${ }^{\circledR}$ was used to filter off the $\mathrm{Pd} / \mathrm{C}$ and was washed with $\mathrm{CH}_{2} \mathrm{Cl}_{2}$. The organic layer was extracted with brine, dried $\left(\mathrm{MgSO}_{4}\right)$ and concentrated under vacuum. The residue was purified by column chromatography on silica gel (hexane/ethyl acetate).

(R)-2-amino-2-(4-methoxyphenyl)-1,1-diphenylethanol (13a). Prepared according to general procedure from (R)-2-(allylamino)-2-(4-methoxyphenyl)-1,1-diphenylethanol 12a (200 $\mathrm{mg}, 0.57$ mmol), methanesulfonic acid $(75 \mu \mathrm{L}, 1.15 \mathrm{mmol}), 10 \% \mathrm{Pd} / \mathrm{C}(79 \mathrm{mg})$ and water $(7 \mathrm{~mL})$ to provide the pure product as a white solid in $61 \%$ yield $(110 \mathrm{mg}, 0.34 \mathrm{mmol}) . \mathrm{Mp}=166{ }^{\circ} \mathrm{C} ;[\alpha]_{\mathrm{D}}+240.8(c 1.04$, $\left.\mathrm{CHCl}_{3}\right) ;{ }^{1} \mathrm{H}-\mathrm{NMR}\left(400 \mathrm{MHz}, \mathrm{CDCl}_{3}\right) \delta 7.74(\mathrm{~d}, J=9 \mathrm{~Hz}, 2 \mathrm{H}), 7.40-7.00(\mathrm{~m}, 10 \mathrm{H}), 6.65(\mathrm{~d}, J=9 \mathrm{~Hz}$, 2H), $4.97(\mathrm{~s}, 1 \mathrm{H}), 3.72(\mathrm{~s}, 3 \mathrm{H}), 1.55$ (broad s, 2H) ppm; ${ }^{13} \mathrm{C}-\mathrm{NMR}\left(100 \mathrm{MHz}, \mathrm{CDCl}_{3}\right) \delta 158.6(\mathrm{C}), 146.6$ (C), $144.0(\mathrm{C}), 132.2(\mathrm{C}), 129.6(\mathrm{CH}), 128.5(\mathrm{CH}), 127.4(\mathrm{CH}), 126.9(\mathrm{CH}), 126.5(\mathrm{CH}), 126.2(\mathrm{CH})$, $126.0(\mathrm{CH}), 112.8(\mathrm{CH}), 79.5(\mathrm{C}), 61.1(\mathrm{CH}), 55.1\left(\mathrm{CH}_{3}\right)$ ppm; IR $(\mathrm{KBr}) v_{\max .}$ 3375, 2904, 2830, 1610, $1514 \mathrm{~cm}^{-1}$. MS $\left.\left(\mathrm{CI}_{\left.-\mathrm{NH}_{3}\right)}\right) \mathrm{m} / \mathrm{z}: 320.1\left[\left(\mathrm{M}^{+}+1\right), 11 \%\right)\right], 135.8\left[\left(\mathrm{C}_{8} \mathrm{H}_{10} \mathrm{NO}^{+}\right), 100 \%\right]$; Anal. Cald for $\mathrm{C}_{21} \mathrm{H}_{21} \mathrm{NO}_{2}$ : C 78.97, H 6.63, N 4.39 Found C 78.98, H 6.43, N 4.25.

(R)-2-amino-2-(4-fluorophenyl)-1,1-diphenylethanol (13b). Prepared according to general procedure from $(R)-2$-(allylamino)-2-(4-fluorophenyl)-1,1-diphenylethanol 12b (100 mg, $0.29 \mathrm{mmol})$, methanesulfonic acid $(37 \mu \mathrm{L}, 0.57 \mathrm{mmol}), 10 \% \mathrm{Pd} / \mathrm{C}(39 \mathrm{mg})$ and water $(4 \mathrm{~mL})$ to provide the pure 
product as a white solid in $74 \%$ yield $(65 \mathrm{mg}, 0.21 \mathrm{mmol}) . \mathrm{Mp}=69-71{ }^{\circ} \mathrm{C} ;[\alpha]_{\mathrm{D}}+190.3 .6(c$ 1.00, $\left.\mathrm{CHCl}_{3}\right) ;{ }^{1} \mathrm{H}-\mathrm{NMR}\left(400 \mathrm{MHz}, \mathrm{CDCl}_{3}\right) \delta 7.74(\mathrm{~d}, J=7 \mathrm{~Hz}, 2 \mathrm{H}), 7.40(\mathrm{t}, J=8 \mathrm{~Hz}, 2 \mathrm{H}), 7.28(\mathrm{t}, J=8 \mathrm{~Hz}$, 2H), 7.13-6.98 (m, 6H), $5.00(\mathrm{~s}, 1 \mathrm{H}), 1.58($ broad s, $2 \mathrm{H}) \mathrm{ppm} ;{ }^{13} \mathrm{C}-\mathrm{NMR}\left(100 \mathrm{MHz}, \mathrm{CDCl}_{3}\right) \delta 161.9(\mathrm{C}$, $\left.J_{F}=244 \mathrm{~Hz}\right), 146.2(\mathrm{C}), 143.7(\mathrm{C}), 135.7(\mathrm{C}), 130.1\left(\mathrm{CH}, J_{F}=8 \mathrm{~Hz}\right), 128.6(\mathrm{CH}), 127.4(\mathrm{CH}), 127.1$ $(\mathrm{CH}), 126.4(\mathrm{CH}), 126.3(\mathrm{CH}), 125.9(\mathrm{CH}), 114.2\left(\mathrm{CH}, J_{F}=21 \mathrm{~Hz}\right), 79.5(\mathrm{C}), 61.0(\mathrm{CH}) \mathrm{ppm}$; IR $(\mathrm{KBr})$ $v_{\max }$ 3370, 3058, 2361, 1652, $1509 \mathrm{~cm}^{-1}$; MS $\left(\mathrm{CI}-\mathrm{NH}_{3}\right) \mathrm{m} / z: 308.1\left[\left(\mathrm{M}^{+}+1,5.6 \%\right), 123.8\left(\mathrm{C}_{7} \mathrm{H}_{7} \mathrm{FN}^{+}\right.\right.$, $100 \%)]$; HRMS (CI-CH 4$)$; calcd for $\mathrm{C}_{20} \mathrm{H}_{18} \mathrm{FNO}\left(\mathrm{M}+\mathrm{H}^{+}\right)$308.1451, found 308.1443.

(R)-2-amino-2-biphenyl-1,1-diphenylethanol (13c). Prepared according to general procedure from (R)-2-(allylamino)-2-biphenyl-1,1-diphenylethanol 12c (75 mg, $0.18 \mathrm{mmol})$, methanesulfonic acid (24 $\mu \mathrm{L}, 0.37 \mathrm{mmol}), 10 \% \mathrm{Pd} / \mathrm{C}(25 \mathrm{mg})$ and water $(5 \mathrm{~mL})$ to provide the pure product as a white solid in 64 $\%$ yield $(39 \mathrm{mg}, 0.11 \mathrm{mmol}) . \mathrm{Mp}=168^{\circ} \mathrm{C} ;[\alpha]_{\mathrm{D}}+241.6\left(c 1.01, \mathrm{CHCl}_{3}\right) ;{ }^{1} \mathrm{H}-\mathrm{NMR}\left(400 \mathrm{MHz}, \mathrm{CDCl}_{3}\right) \delta$ $7.76(\mathrm{~d}, J=8 \mathrm{~Hz}, 2 \mathrm{H}), 7.53(\mathrm{~d}, J=8 \mathrm{~Hz}, 2 \mathrm{H}), 7.44-6.95(\mathrm{~m}, 15 \mathrm{H}), 5.06(\mathrm{~s}, 1 \mathrm{H}), 4.66($ broad s, $1 \mathrm{H}), 1.55$ (broad s, 2H) ppm; ${ }^{13} \mathrm{C}-\mathrm{NMR}\left(100 \mathrm{MHz}, \mathrm{CDCl}_{3}\right) \delta 146.4$ (C), 143.8 (C), 140.7 (C), 139.9 (C), 139.2 (C), $129.0(\mathrm{CH}), 128.6(\mathrm{CH}), 128.5(\mathrm{CH}), 127.4(\mathrm{CH}), 127.1(\mathrm{CH}), 127.0(\mathrm{CH}), 126.9(\mathrm{CH}), 126.5$ $(\mathrm{CH}), 126.3(\mathrm{CH}), 126.0(\mathrm{CH}) \mathrm{ppm}$; IR $(\mathrm{KBr}) v_{\max } 3379,3031,1489,1448,746 \mathrm{~cm}^{-1}$; MS $\left(\mathrm{CI}-\mathrm{NH}_{3}\right) \mathrm{m} / z$ : $366.2\left[\left(\mathrm{M}^{+}+1\right), 57 \%, 182.0\left(\mathrm{C}_{13} \mathrm{H}_{12} \mathrm{~N}^{+}\right), 100 \%\right]$; Anal. Cald for $\mathrm{C}_{26} \mathrm{H}_{23} \mathrm{NO}$ : C 85.89, H 6.71, N 3.45 Found C 86.20, H 6.37, N 3.35.

(R)-2-amino-2-(3,5-dimethylphenyl)-1,1-diphenylethanol (13d). Prepared according to general procedure from (R)-2-(allylamino)-2-(3,5-dimethylphenyl)-1,1-diphenylethanol 12d (71 mg, 0.20 $\mathrm{mmol})$, methanesulfonic acid $(26 \mu \mathrm{L}, 0.40 \mathrm{mmol}), 10 \% \mathrm{Pd} / \mathrm{C}(30 \mathrm{mg})$ and water $(3 \mathrm{~mL})$ to provide the pure product as a white solid in $66 \%$ yield $(38 \mathrm{mg}, 0.12 \mathrm{mmol}) . \mathrm{Mp}=132-135^{\circ} \mathrm{C} ;[\alpha]_{\mathrm{D}}+141.4(c 1.00$, $\left.\mathrm{CHCl}_{3}\right) ;{ }^{1} \mathrm{H}-\mathrm{NMR}\left(400 \mathrm{MHz}, \mathrm{CDCl}_{3}\right) \delta 7.73(\mathrm{~d}, J=8 \mathrm{~Hz}, 2 \mathrm{H}), 7.39(\mathrm{t}, J=8 \mathrm{~Hz}, 2 \mathrm{H}), 7.27(\mathrm{t}, J=7 \mathrm{~Hz}$, 2H), 7.13-6.98 (m, 4H), $6.76(\mathrm{~s}, 1 \mathrm{H}), 6.69$ (s, 2H), 4.92 (s, 1H), 2.14 (s, 6H); ${ }^{13} \mathrm{C}-\mathrm{NMR}(100 \mathrm{MHz}$, 
$\left.\mathrm{CDCl}_{3}\right) \delta 146.6(\mathrm{C}), 144.1(\mathrm{C}), 140.0(\mathrm{C}), 136.9(\mathrm{C}), 128.8(\mathrm{CH}), 128.6(\mathrm{CH}), 127.4(\mathrm{CH}), 127.1(\mathrm{CH})$, $126.7(\mathrm{CH}), 126.6(\mathrm{CH}), 126.4(\mathrm{CH}), 126.3(\mathrm{CH}), 79.7(\mathrm{C}), 61.8(\mathrm{CH}), 21.3\left(\mathrm{CH}_{3}\right) \mathrm{ppm}$; IR $(\mathrm{KBr}) v_{\max }$

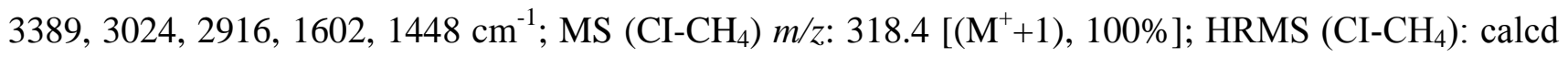
for $\mathrm{C}_{22} \mathrm{H}_{23} \mathrm{NO}\left(\mathrm{M}+\mathrm{H}^{+}\right) 318.1858$, found 318.1857 . 


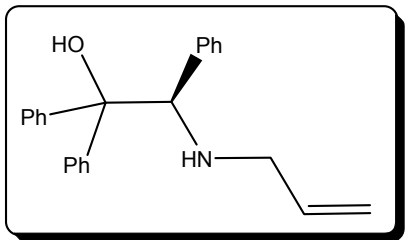

${ }^{1} \mathrm{H}-\mathrm{NMR}\left(400 \mathrm{MHz}, \mathrm{CDCl}_{3}\right.$ )

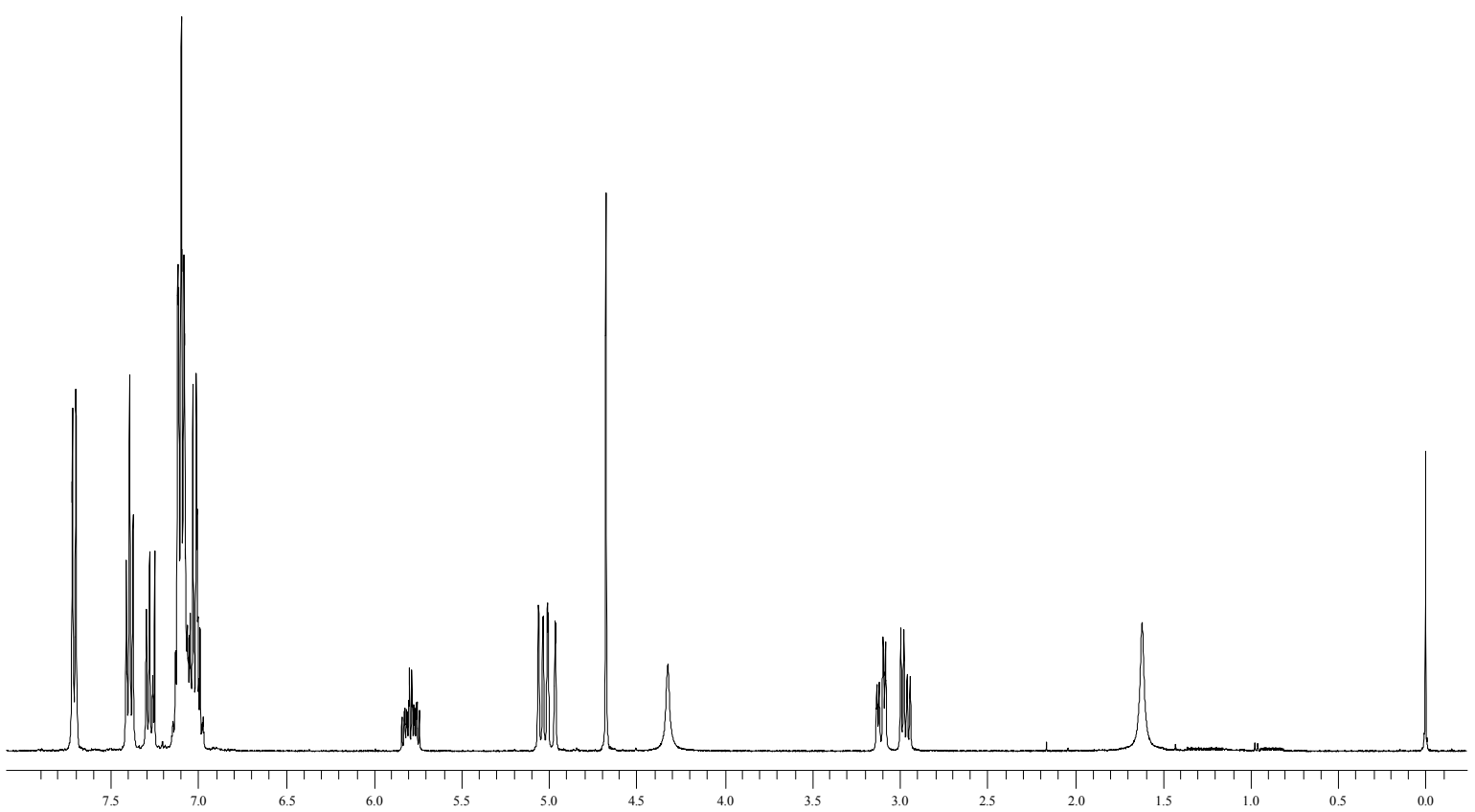

${ }^{13} \mathrm{C}-\mathrm{NMR}\left(100 \mathrm{MHz}, \mathrm{CDCl}_{3}\right)$

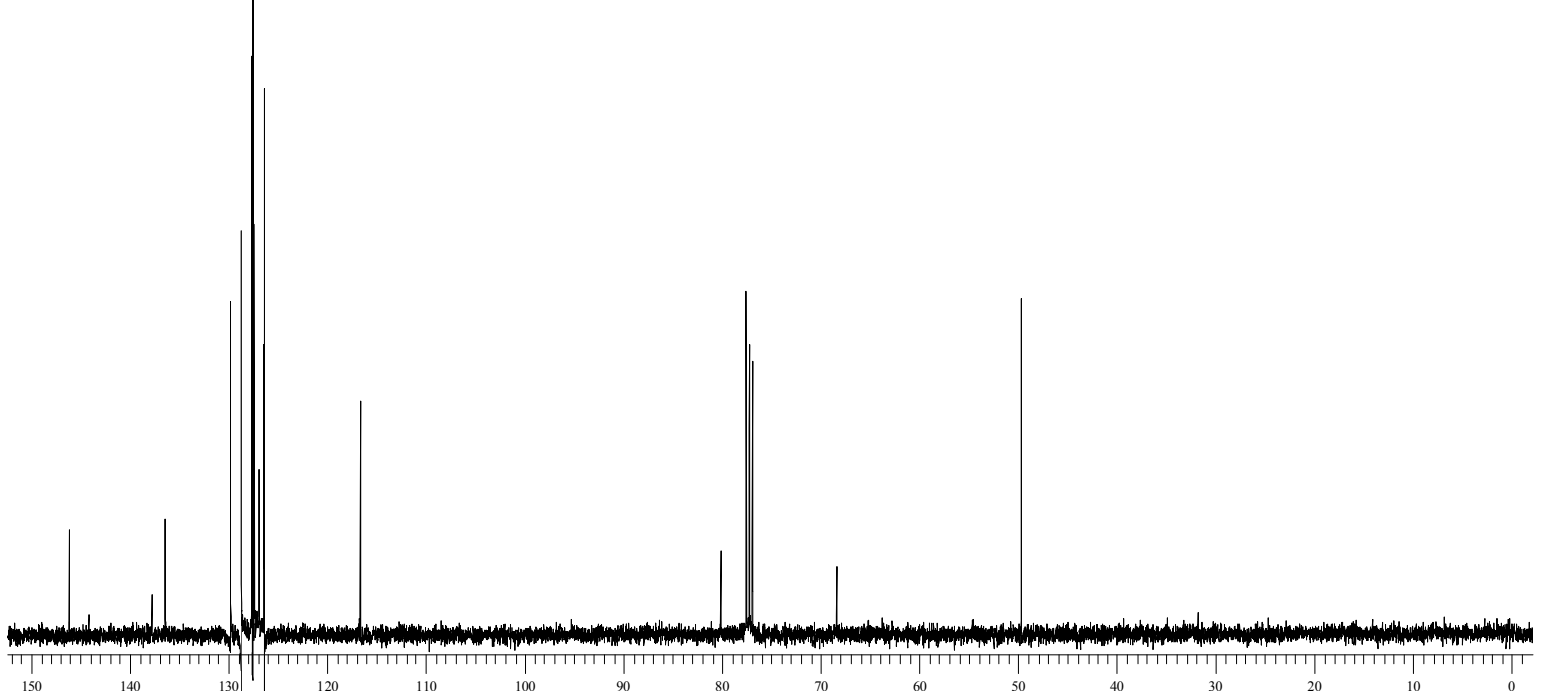




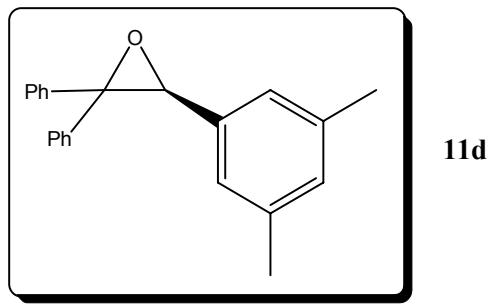

${ }^{1} \mathrm{H}-\mathrm{NMR}\left(400 \mathrm{MHz}, \mathrm{CDCl}_{3}\right.$ )

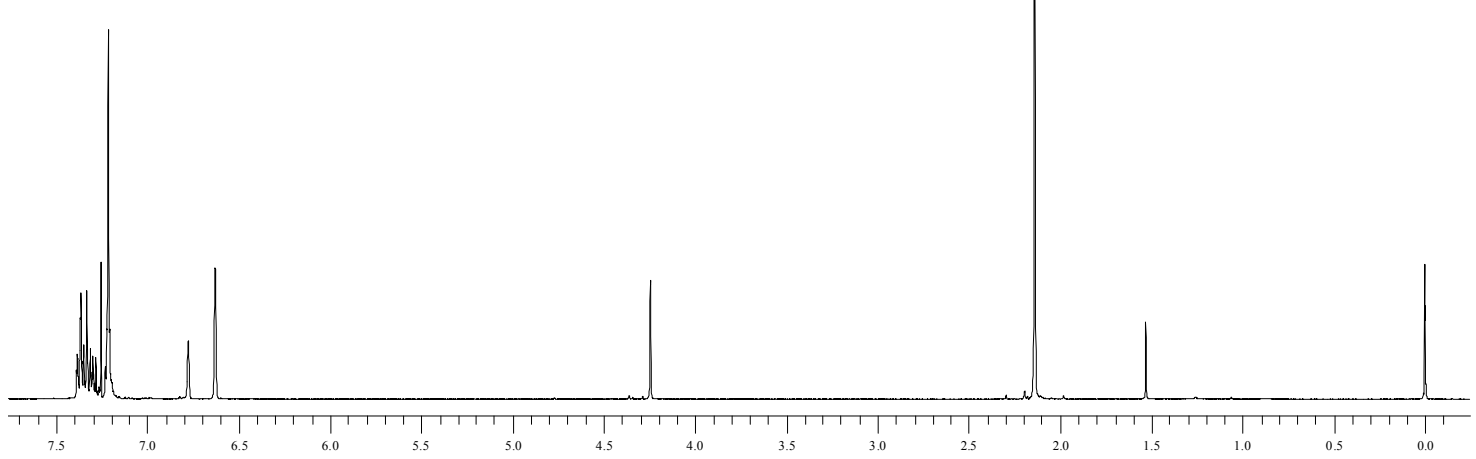

${ }^{13} \mathrm{C}-\mathrm{NMR}\left(100 \mathrm{MHz}, \mathrm{CDCl}_{3}\right)$

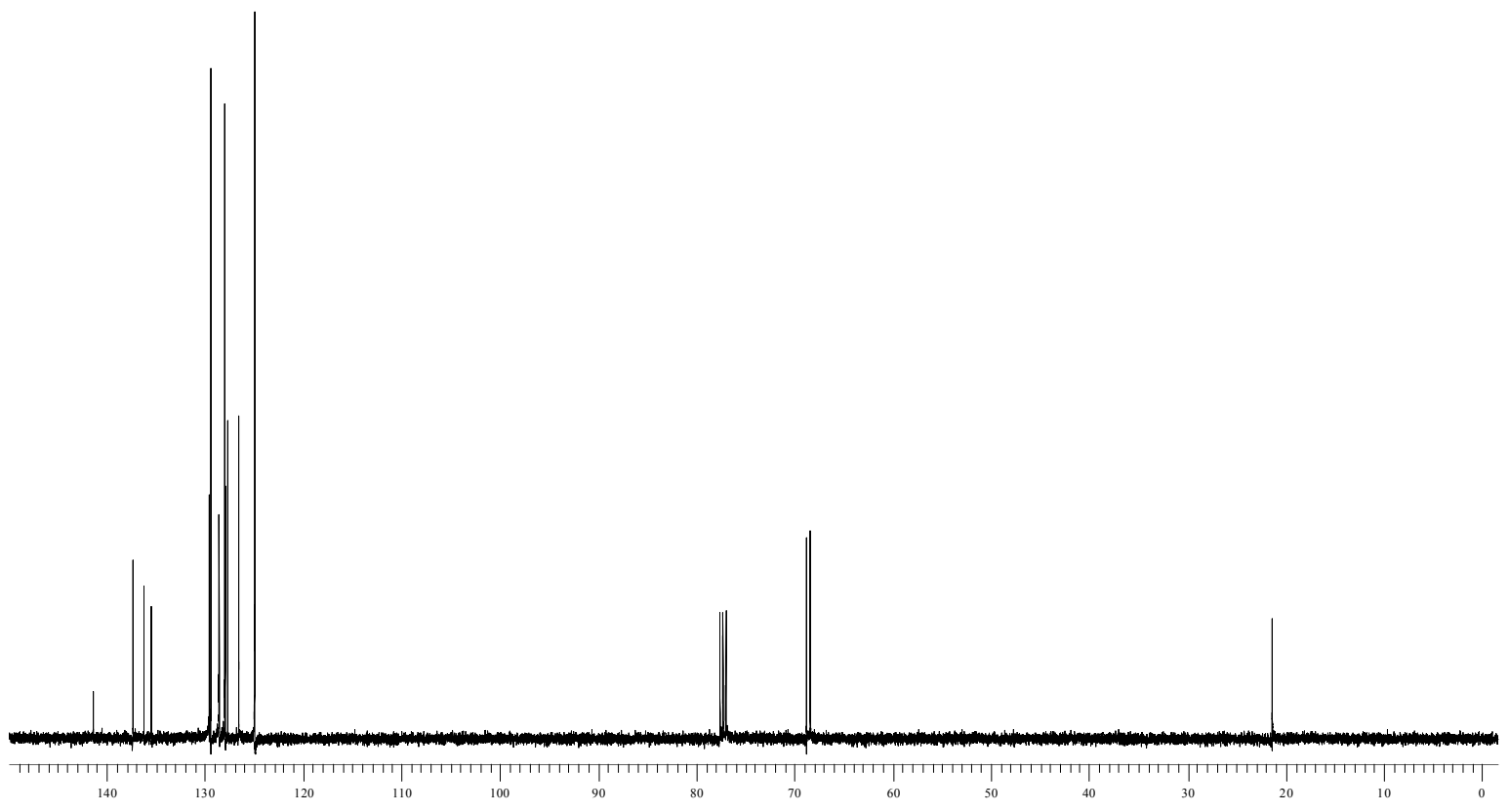




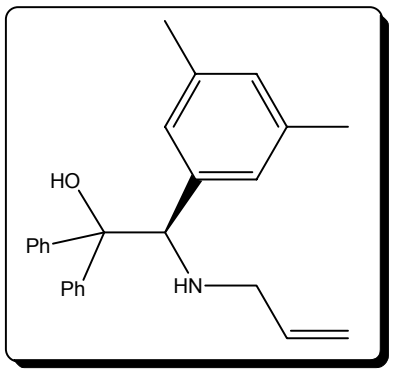

${ }^{1} \mathrm{H}-\mathrm{NMR}\left(400 \mathrm{MHz}, \mathrm{CDCl}_{3}\right.$ )

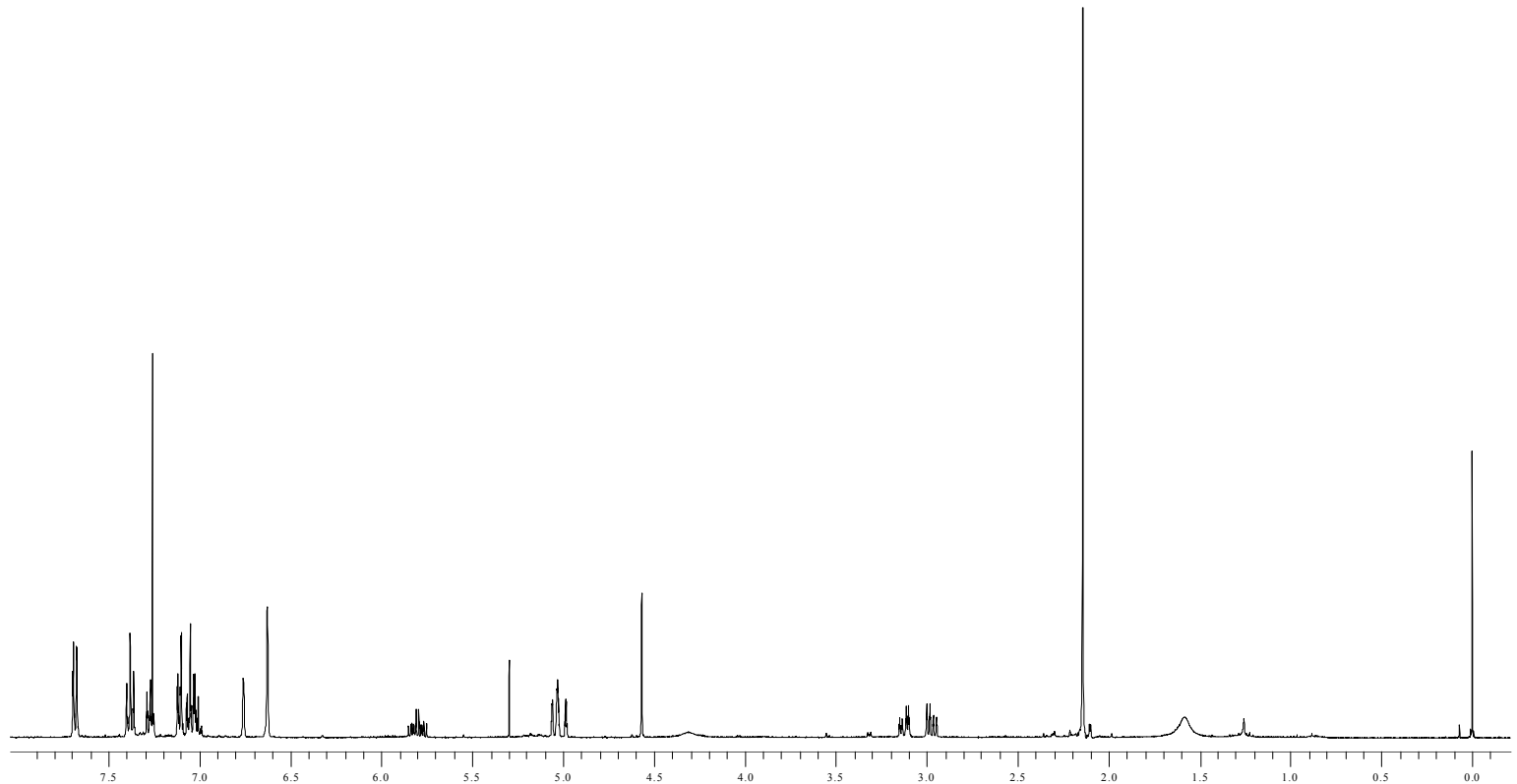

${ }^{13} \mathrm{C}-\mathrm{NMR}\left(100 \mathrm{MHz}, \mathrm{CDCl}_{3}\right)$

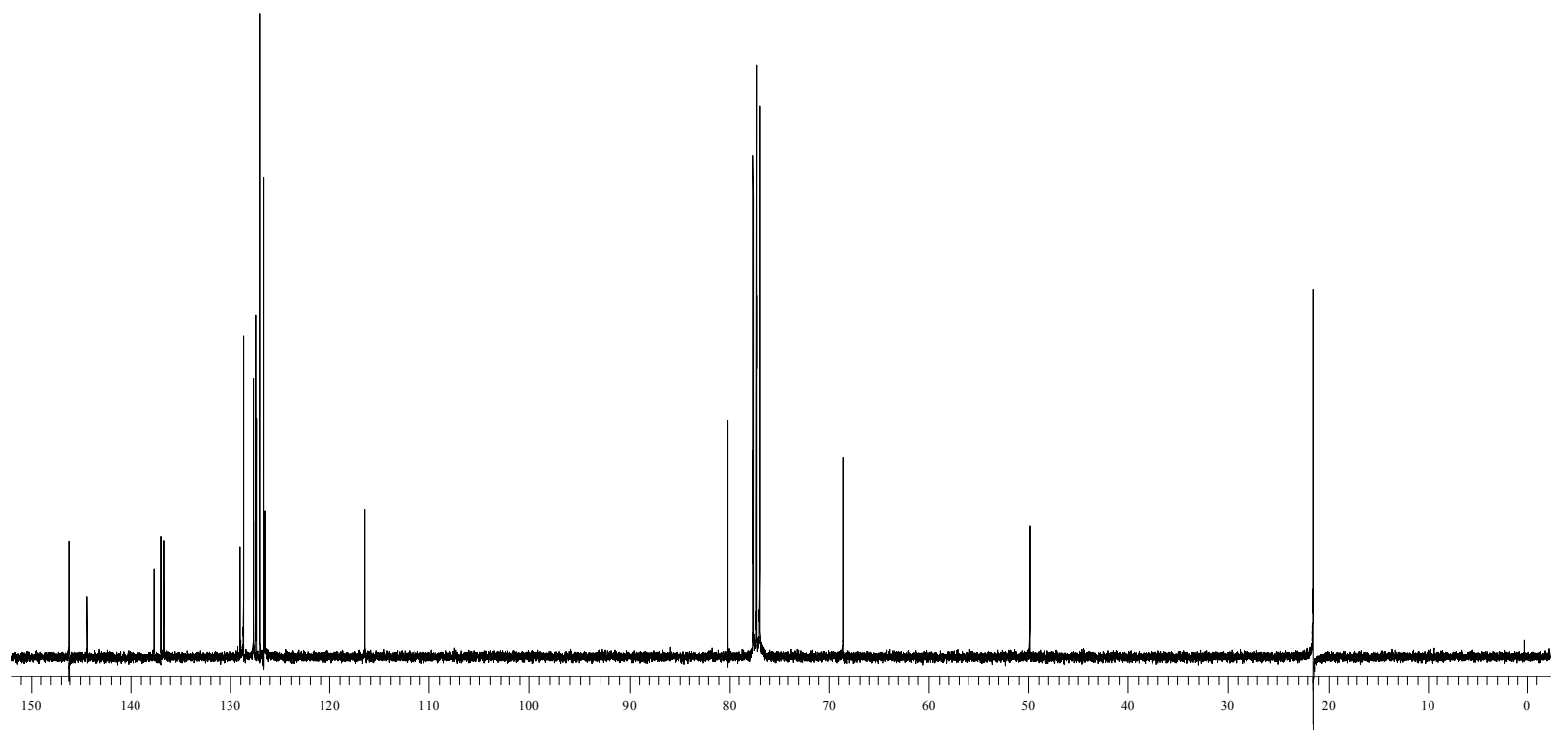




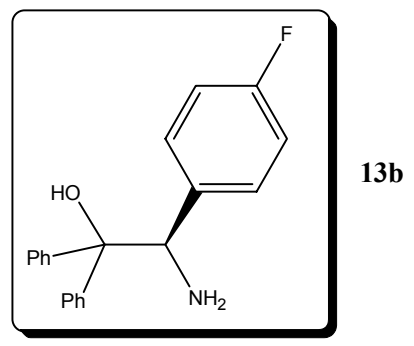

${ }^{1} \mathrm{H}-\mathrm{NMR}\left(400 \mathrm{MHz}, \mathrm{CDCl}_{3}\right.$ )

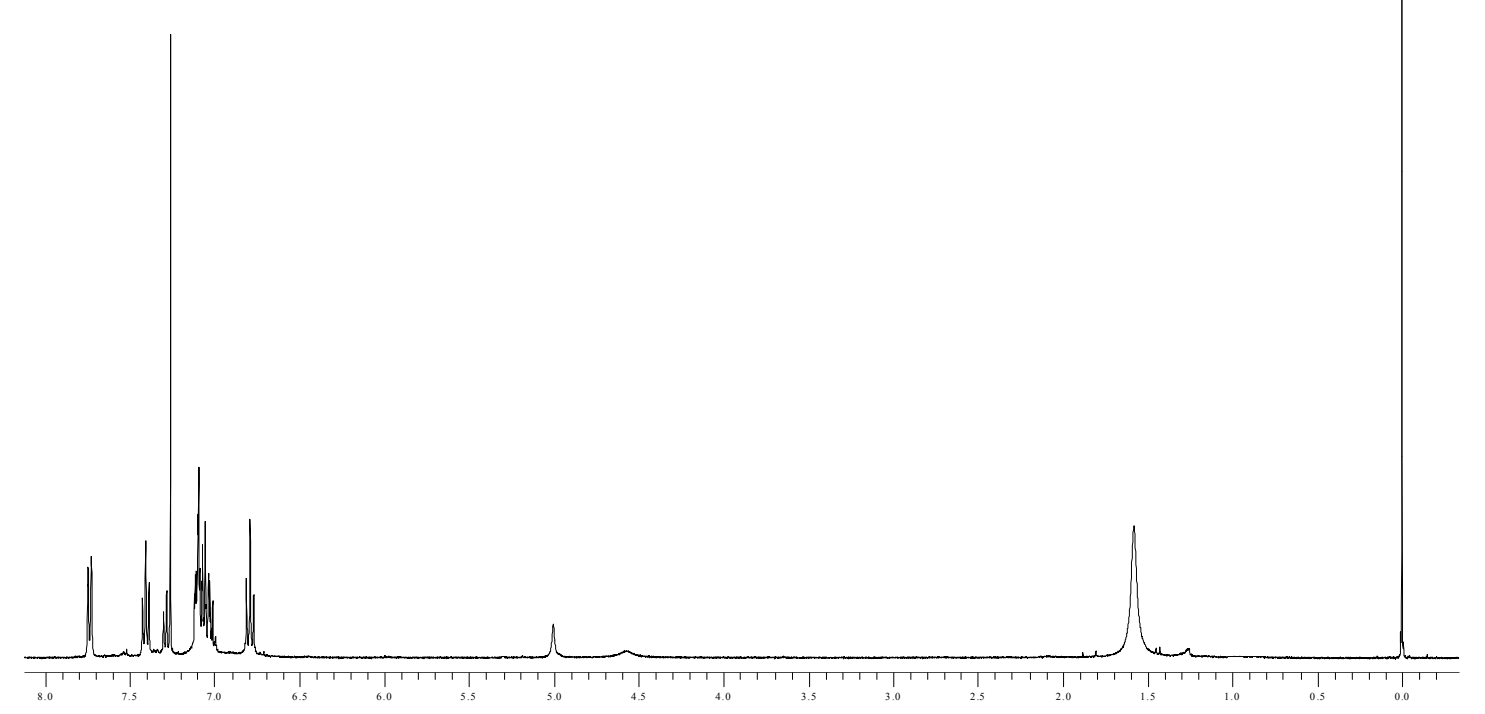

${ }^{13} \mathrm{C}-\mathrm{NMR}\left(100 \mathrm{MHz}, \mathrm{CDCl}_{3}\right.$ )

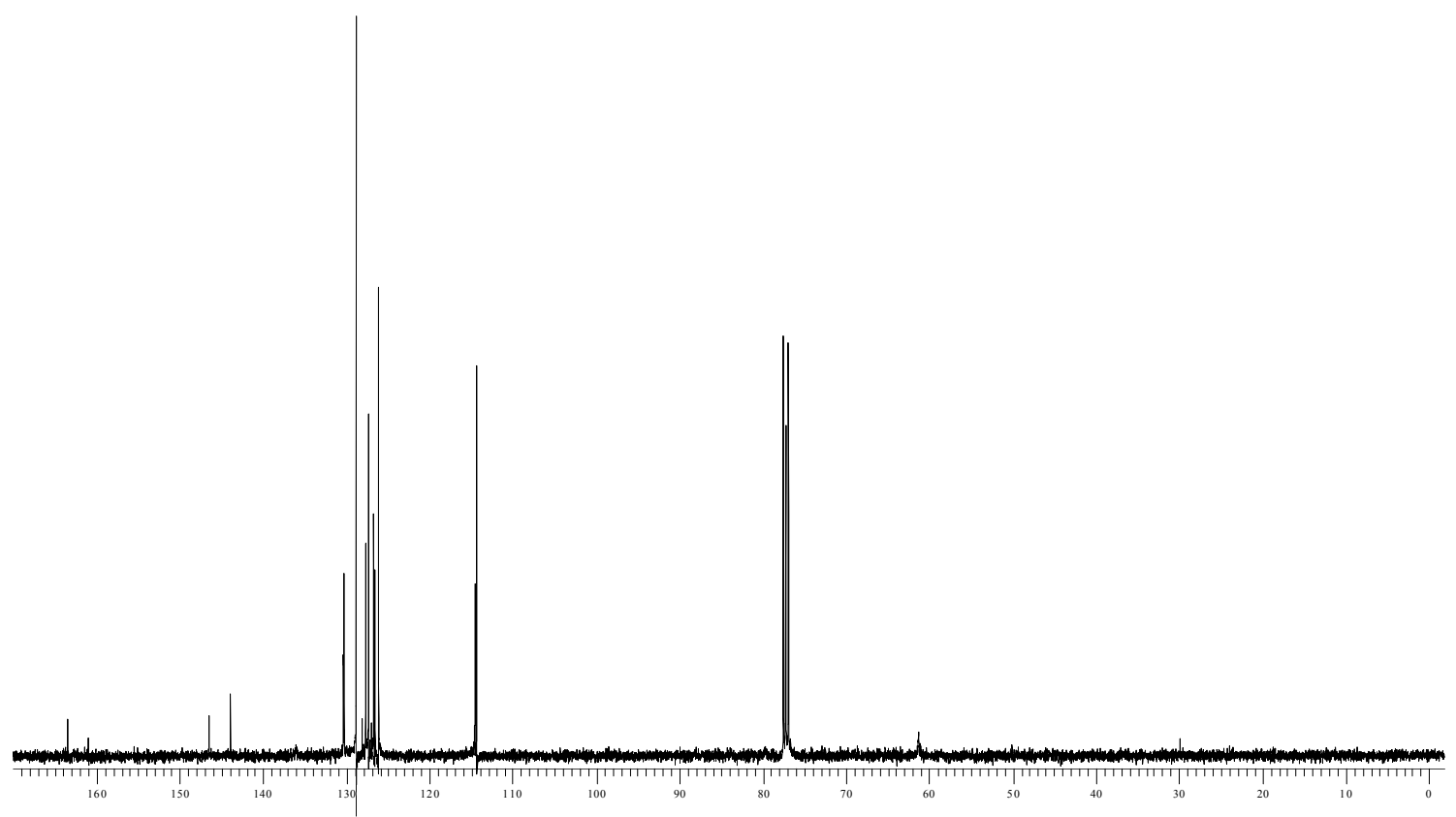




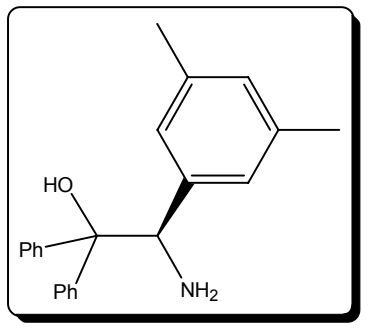

${ }^{1} \mathrm{H}-\mathrm{NMR}\left(400 \mathrm{MHz}, \mathrm{CDCl}_{3}\right.$ )

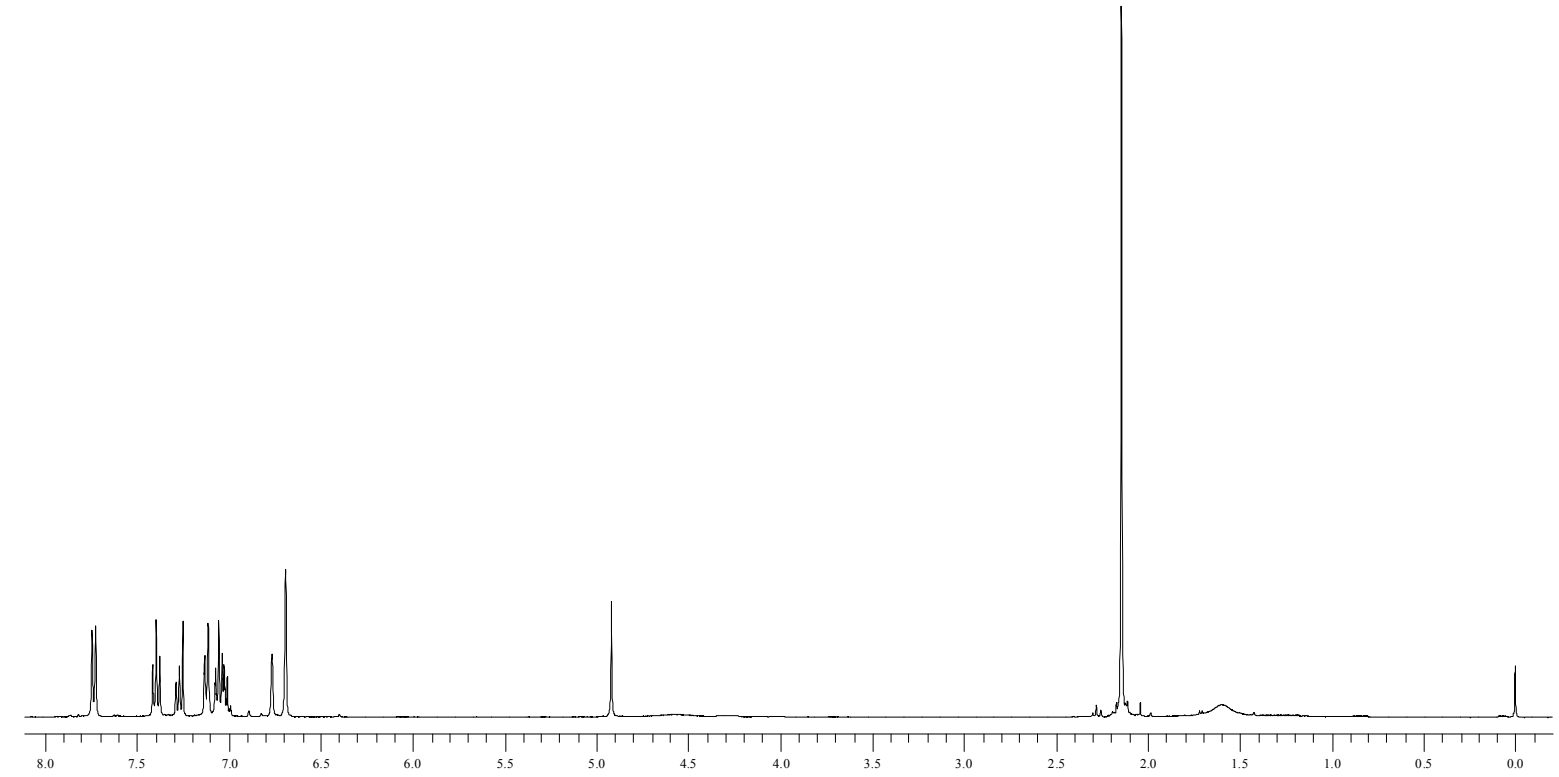

${ }^{13} \mathrm{C}-\mathrm{NMR}\left(100 \mathrm{MHz}, \mathrm{CDCl}_{3}\right.$ )

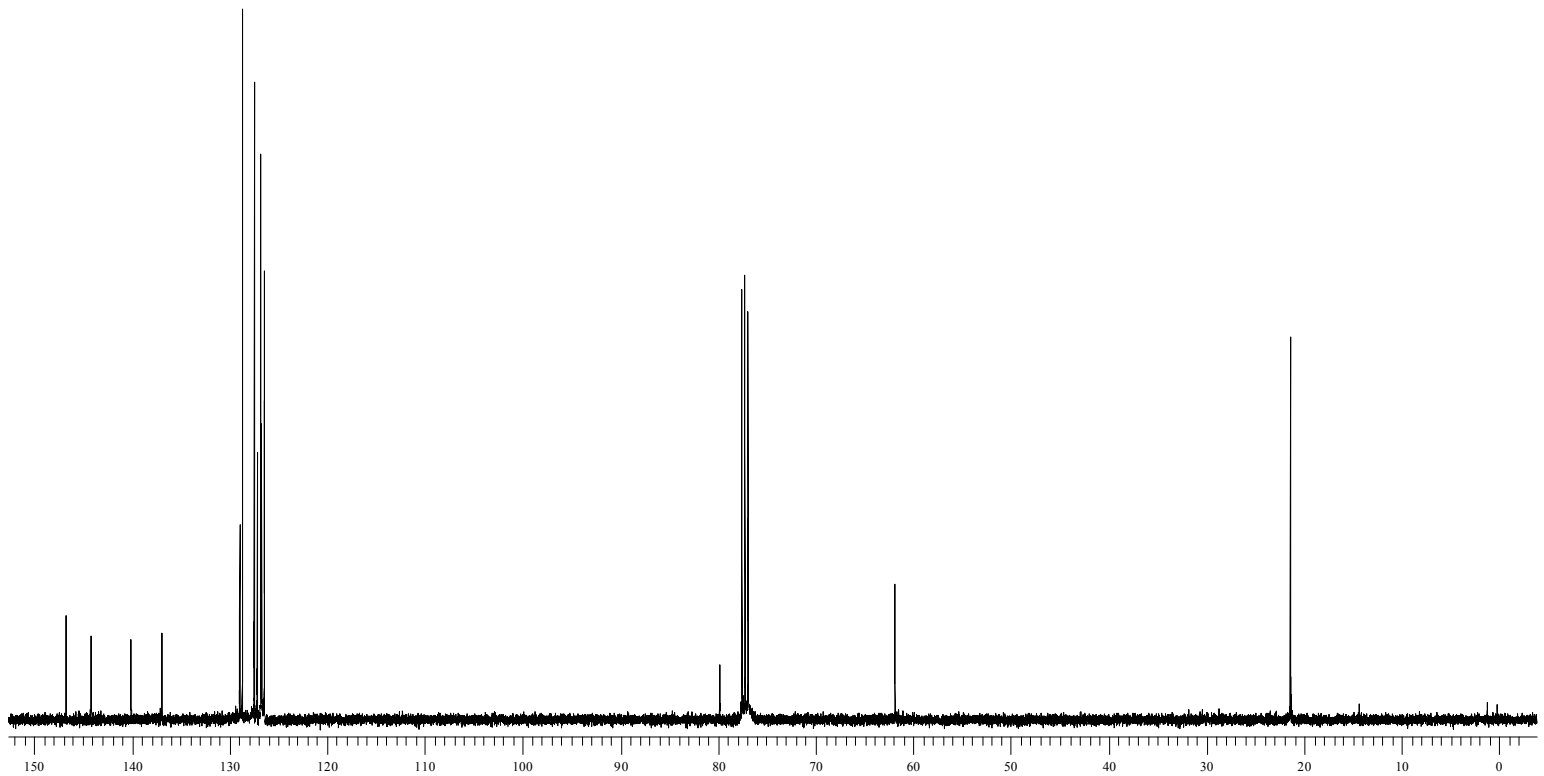

